\title{
Energy identity for a class of approximate harmonic maps from surfaces
}

\author{
Weiyue Ding and Gang Tian $^{1}$
}

\section{Introduction.}

Consider the heat equation for harmonic maps from a closed Riemannian surface $(M, g)$ into a closed submanifold $N$ in $R^{K}$ :

$$
\frac{\partial u}{\partial t}=\tau_{g}(u)
$$

where

$$
\tau_{g}(u)=\Delta_{g} u+g^{\alpha, \beta} A(u)\left(u, \alpha, u_{, \beta}\right),
$$

and $A(u)$ is the 2 nd fundamental form of $N$ in $R^{K}$. Let $u: M \times(0, \infty) \rightarrow N$ be a global weak solution to (1.1). (See [St].) Then $u$ is smooth away from a finite number of singular points $\left\{\left(p_{i}, T_{i}\right)\right\} \subset M \times(0, \infty)$. Let $(p, T)$ be a singular point. Then we may assume that for some $t_{0} \in(0, T)$ and a neighborhood $U$ of $p, u$ is smooth on $\bar{U} \times\left[t_{0}, T\right)$ and $u(t)=u(\cdot, t) \rightarrow$ $u(T)=u(\cdot, T)$ smoothly on $\bar{U} \backslash\{p\}$ as $t$ tends to $T$ from left. Moreover, by choosing a suitable sequence $t_{i} \rightarrow T$ and by rescaling $u\left(t_{i}\right)$ properly near $p$, one can show that there exist finitely many "bubbles" (i.e. harmonic maps $\left.\varphi_{j}: S^{2} \rightarrow N, 1 \leq j \leq m,\right)$ associated with the sequence $\left\{u\left(t_{i}\right)\right\}$. These bubbles are responsible for the loss of energy and we have

$$
\lim _{t_{i} \rightarrow T} E_{U}\left(u\left(t_{i}\right)\right)-E_{U}(u(T)) \geq \sum_{j=1}^{m} E\left(\varphi_{j}\right) .
$$

It is generally believed that the above inequality should be an identity, which we will call the "energy identity". Since it can be easily shown that the limit

$$
\lim _{t \rightarrow T-0} E_{U}(u(t))
$$

${ }^{1}$ This work is supported in part by the National Natural Science Fundation of China. The second author is also partailly supported by NSF grant. 
exists, we may write the energy identity as

$$
\lim _{t \rightarrow T-0} E_{U}(u(t))=E_{U}(u(T))+\sum_{j=1}^{m} E\left(\varphi_{j}\right) .
$$

(But note that we do not know if the set $\left\{\varphi_{j}\right\}$ of "bubbles" is unique, i.e. there is some possibility that it may depend on the specific time sequence $t_{i}$.) The identity (1.2) means that "blowing bubbles" is the only reason for the energy loss. J. Qing [Q] proved that (1.2) holds in the special case where $N$ is the standard sphere $S^{n}$. In this note we will give a simple proof of (1.2) for the general targets $N$.

If we consider the solution $u(t)$ of (1.1) as maps which in some sense approximate harmonic maps, we must have certain control of the tension field $\tau(u(t))$. We will see that a good control is the boundedness of the $L^{2}$ norm, i.e.

$$
\left\|\tau\left(u\left(t_{i}\right)\right)\right\|_{L^{2}(M)} \leq C
$$

for some sequence $t_{i} \rightarrow T$. However, from Eq. (1.1) we can only get

$$
\int_{t_{0}}^{T}\|\tau(u(t))\|_{L^{2}(M)}^{2} d t<\infty .
$$

Since $T<\infty$, there is no guarantee for the existence of $t_{i} \rightarrow T$ such that (1.3) holds. On the other hand, if $T=\infty$ then such a sequence is easy to get. Therefore, our analysis in the following easily leads to the energy identity for blow-ups at time-infinity. More precisely, we have

Theorem 1. Let $u$ be a global weak solution to (1.1) and $t_{i} \rightarrow \infty$ be a sequence such that $u\left(t_{i}\right) \rightarrow u_{\infty}$ weakly in $W^{1,2}\left(M, R^{K}\right)$ and (1.3) holds. Suppose that the convergence $u\left(t_{i}\right) \rightarrow u_{\infty}$ is not $W^{1,2}$-strong. Then there exits a subsequence, still denoted by $u\left(t_{i}\right)$, and a finite set of harmonic maps $\varphi_{j}: S^{2} \rightarrow N(j=1, \cdots, m)$ such that

$$
\lim _{t_{i} \rightarrow \infty} E\left(u\left(t_{i}\right)\right)=E\left(u_{\infty}\right)+\sum_{j=1}^{m} E\left(\varphi_{j}\right) .
$$

Note that the limit on the left does not depend on the specific sequence $u\left(t_{i}\right)$, but $u_{\infty}$ and the set $\left\{\varphi_{j}\right\}$ may depend on it. Theorem 1 follows easily from the following 
Theorem 2. Let $u_{i}$ be a squence of maps in $W^{2,2}(D, N)$ with bounded energy $E\left(u_{i}\right) \leq C$, where $D$ is the unit disk. Assume that (i) $u_{i}$ converge strongly to $u_{0}$ in $W_{l o c}^{1,2}(D \backslash\{0\}, N)$, but the convergence is not $W^{1,2}(D, N)$ strong; and (ii) the $L^{2}$-norms of the tension fields $\tau\left(u_{i}\right)$ are uniformly bounded. Then there exists a subsequence, still denoted by $u_{i}$, and a finite set of harmonic maps $\varphi_{j}: S^{2} \rightarrow N(j=1, \cdots, m)$ such that

$$
\lim _{i \rightarrow \infty} E\left(u_{i}\right)=E\left(u_{0}\right)+\sum_{j=1}^{m} E\left(\varphi_{j}\right) .
$$

The derivation of (1.2) from Theorem 2 needs more work. By an argument of Qing (see $\S 2$ in [Q] ), it can be shown that Theorem 2 implies that the energy identity (1.2) holds true, and hence we have

Theorem 2. Let $u$ be a global weak solution to (1.1) and $T \in(0, \infty)$. Assume that $u(t) \rightarrow u(T)$ weakly in $W^{1,2}\left(M, R^{K}\right)$, but the convergence is not $W^{1,2}$-strong. Then there exists a finite set of harmonic maps $\varphi_{j}: S^{2} \rightarrow N$ $(j=1, \cdots, m)$ such that

$$
\lim _{t \rightarrow T-0} E(u(t))=E(u(T))+\sum_{j=1}^{m} E\left(\varphi_{j}\right) .
$$

We should mention that when $u_{i}$ is a sequence of harmonic maps (i.e. $\tau\left(u_{i}\right)=0$ ), Theorem 2 was first proved by J. Jost in [J] ( Lemma 4.3.1, p.127). More recently, T. Parker proved in $[\mathrm{P}]$ that if the $u_{i}$ is a sequence of harmonic maps, the limit $u_{0} \cup_{j}\left\{\varphi_{j}\right\}$ maps to a connected set.

Part of this work was done when the second author was at Peking Uni-. versity in the summer of 1994. After we finished the writing of this paper, we learned from F. H. Lin that L. B. Mou and C. Y. Wang also obtained results similar to ours, and their methods are different from ours.

\section{Proof of Theorem 2.}

Let $u_{i} \in W^{2,2}(D, N)$ be a sequence of maps which satisfies the conditions of Theorem 2. Before proving the energy identity (1.4) we need some preliminaries. The following estimate is similar to the main estimate in [S-U] and will be important for our analysis.

Lemma 2.1. Let $u \in W^{2, p}(D, N), 1<p \leq 2$. There exists $\epsilon_{0}>0$ such that if $E(u)<\epsilon_{0}$ then

$$
\|u-\bar{u}\|_{W^{2, p}\left(D_{1 / 2}\right)} \leq C\left[\|\nabla u\|_{L^{p}(D)}+\|\tau(u)\|_{L^{p}(D)}\right],
$$


where $\bar{u}$ is the mean value of $u$ over the unit disk $D$.

Proof. It will be convenient to assume that $\bar{u}=0$. Then we have the inequality

$$
\|u\|_{0, p} \leq C_{p}\|\nabla u\|_{0, p} .
$$

Here and in the following, we use $\|\cdot\|_{k, p}$ to denote the $W^{k, p}(D)$-norm. Consider $u$ as a solution to the equation

$$
\Delta u+A(u)(d u, d u)=\alpha,
$$

where $\alpha=\tau(u) \in L^{p}(D)$. Assume first that $1<p<2$. Let $\phi$ be a cut-off function which equals 1 on $D_{3 / 4}, 0$ on $\partial D$, and $0 \leq \phi \leq 1$ otherwise. From the equation we get that

$$
|\Delta(\phi u)| \leq C(|\nabla(\phi u)||\nabla u|+|\nabla u|+|u|+|\alpha|) .
$$

By the standard $L^{p}$-estimate we have

$$
\|\phi u\|_{2, p} \leq C\left(\left\|\left|\nabla(\phi u)\|\nabla u \mid\|_{0, p}+\|\nabla u\|_{0, p}+\|\alpha\|_{0, p}\right) .\right.\right.
$$

But

$$
\left\|\left|\nabla(\phi u)\|\nabla u \mid\|_{0, p} \leq\|\nabla(\phi u)\|_{0, q}\|\nabla u\|_{0,2},\right.\right.
$$

where $q=2 p /(2-p)$. Since we have the Sobolev's imbedding $W^{2, p} \subset W^{1, q}$, so

$$
\|\phi u\|_{2, p} \leq C\left(E(u)^{1 / 2}\|\phi u\|_{2, p}+\|\nabla u\|_{0, p}+\|\alpha\|_{0, p}\right) .
$$

Thus, if $E(u, D)$ is small, (2.1) follows.

Next, if $p=2$, one can first derive the above estimate with $p=4 / 3$. Such an estimate gives a $L^{4}\left(D_{3 / 4}\right)$-bound for $|\nabla u|$. Then one can apply the interior $L^{2}$-estimate to the equation for $u$ and get (2.1) with $p=2$.

Remark. We will only need the case $p=2$ in Lemma 2.1. But note that even for $1<p<2$, the lemma provides the necessary compactness in the blow-up analysis of Sacks-Uhlenbeck, since the imbedding $W^{2, p} \subset W^{1,2}$ is compact. For instance, if $u_{i}: D \rightarrow N$ is a sequence of $W^{2, p}$ maps with $E\left(u_{i}\right)<\epsilon_{0}$ and $\tau\left(u_{i}\right) \rightarrow 0$ in $L^{P}(D)$, then we see that $u_{i}$ subconverges strongly in $W^{1,2}\left(D_{1 / 2}\right)$ to some harmonic map.

Now we want to prove for certain subsequence of $u_{i}$, still denoted by $u_{i}$, the energy identity

$$
\lim _{i \rightarrow \infty} E\left(u_{i}\right)=E\left(u_{0}\right)+\sum_{j=1}^{m} E\left(\phi_{j}\right)
$$


where $\varphi_{j}: S^{2} \rightarrow N$ are harmonic spheres obtained by suitable rescaling of the sequence $u_{i}$ and taking the limits. Since $u_{i}$ converge strongly in $W^{1,2}\left(D \backslash D_{\delta}, N\right)$ for any $\delta \in(0,1)$, we see that $(2.2)$ is equivalent to

$$
\lim _{\delta \rightarrow 0} \lim _{i \rightarrow \infty} E\left(u_{i}, D_{\delta}\right)=\sum_{j=1}^{m} E\left(\varphi_{j}\right) .
$$

For simplicity we assume that the first bubble is obtained by the scaling as follows. For some $0<\lambda_{i} \rightarrow 0$ and $x_{i} \rightarrow 0$, let

$$
\tilde{u}_{i}(x)=u_{i}\left(x_{i}+\lambda_{i}\left(x-x_{i}\right)\right) .
$$

By choosing $\lambda_{i}$ properly we may assume that $\tilde{u}_{i}$ converges strongly in $W^{1,2}\left(D_{R}\right)$, for every $R \geq 1$, to a harmonic map $\psi_{1}: R^{2} \rightarrow N$ with finite energy. Then $\varphi_{1}=\pi^{*} \psi_{1}$ is the first harmoic sphere with the same energy as $\psi_{1}$, where $\pi: S^{2} \backslash\{N\} \rightarrow R^{2}$ is the stereoprojection. If for given $\delta>0$ small and $R>0$ large, we set

$$
A(\delta, R, i)=\left\{x \in R^{2}: \lambda_{i} R \leq\left|x-x_{i}\right| \leq \delta\right\},
$$

then it is easy to see that (2.3) is equivalent to

$$
\lim _{R \rightarrow \infty} \lim _{\delta \rightarrow 0} \lim _{i \rightarrow \infty} E\left(u_{i}, A(\delta, R, i)\right)=\sum_{j=2}^{m} E\left(\varphi_{j}\right)
$$

It will be convenient to make a conformal change of the domain. Let $\left(r_{i}, \theta_{i}\right)$ be the polar coordinates centered at $x_{i}$. Let $f: R^{1} \times S^{1} \rightarrow R^{2}$ be the. mapping given by $r_{i}=e^{-t}, \theta_{i}=\theta$ for $(t, \theta) \in R^{1} \times S^{1}$. Let $R^{1} \times S^{1}$ be given the product metric $g=d t^{2}+d \theta^{2}$. Then $g$ is conformal to the Euclidean metric $d s^{2}$. In fact, we have

$$
\left(f^{-1}\right)^{*} g=\frac{1}{r_{i}^{2}} d s^{2} .
$$

Fix a large number $R>0$ and a small number $\delta>0$, we will be concerned with the energy of $u_{i}$ on $A(\delta, R, i)$. Let $v_{i}=f^{*} u_{i}$. Then

$$
E\left(u_{i}, A(\delta, R, i)\right)=E\left(v_{i}, B_{i}\right)
$$

where

$$
B_{i}=\left[|\log \delta|,\left|\log \left(\lambda_{i} R\right)\right|\right] \times S^{1} .
$$

We list some important properties of $v_{i}$ inherited from $u_{i}$. 
(1) We will consider $v_{i}:(|\log \delta|, \infty) \times S^{1} \rightarrow N$ as a solution to the equation

$$
\Delta v_{i}+A\left(v_{i}\right)\left(d v_{i}, d v_{i}\right)=\alpha_{i}
$$

where $\alpha_{i}=\tau\left(v_{i}\right)$. Note that

$$
\left\|\tau\left(v_{i}\right)\right\|_{L^{2}}^{2}=\int_{D_{\delta}\left(x_{i}\right)} r_{i}^{2}\left|\tau\left(u_{i}\right)\right|^{2} d x \leq 2 \delta^{2} C .
$$

Thus, $\alpha_{i}$ has very small $L^{2}$-norm if only $\delta$ is very small. On the other hand, since the $L^{1}$-norm of the tension field is conformally invariant, the $L^{1}$-norm of $\alpha_{i}$ is also very small.

(2) For any mapping $u \in W^{2,2}(D, N)$, we may define a "quadratic differential" $\Phi(u)=\phi(u) d z^{2}$ by

$$
\phi(u)=\left|u_{x}\right|^{2}-\left|u_{y}\right|^{2}-2 \sqrt{-1} u_{x} \cdot u_{y} .
$$

It is well known that if $u$ is harmonic then $\Phi(u)$ is holomorphic, i.e. $\bar{\partial} \phi(u)=$ 0 . In fact, if $u \in W^{2, p}$ this can be checked directly by differentiation. One finds that

$$
\bar{\partial} \phi(u)=\left(u_{x}-\sqrt{-1} u_{y}\right) \cdot \tau(u) .
$$

So for our maps $u_{i}$ we have

$$
\left|\bar{\partial} \phi\left(u_{i}\right)\right| \leq\left|\nabla u_{i}\right|\left|\tau\left(u_{i}\right)\right| .
$$

It follows that

$$
\left\|\bar{\partial} \phi\left(u_{i}\right)\right\|_{L^{1}} \leq E\left(u_{i}\right)^{1 / 2}\left\|\tau\left(u_{i}\right)\right\|_{L^{2}} \leq C .
$$

Writing $\phi\left(u_{i}\right)=\phi_{i}$, we have

$$
2 \pi \rho \sqrt{-1} \phi_{i}(z)=\int_{\partial D_{\rho}} \frac{\phi_{i}(\theta)}{\theta-z} d \theta-\int_{D_{\rho}} \frac{\bar{\partial} \phi_{i}(\xi)}{\xi-z} d \xi
$$

where $\rho$ can be any number in $(0,1]$. We choose $\rho=3 / 4$. Note that by assumption, $u_{i} \rightarrow u_{0}$ strongly in $W^{1,2}$. Hence the energy of $u_{i}$ is locally small in a neighborhood $U$ of $\partial D_{\rho}$, and we may apply Lemma 2.1 to conclude that $u_{i}$ have uniformly bounded $W^{2,2}$-norms on $U$. Thus, by the imbedding theorem for trace spaces we see that $\left|\phi_{i}\right|$ are uniformly bounded in $L^{1}\left(\partial D_{\rho}\right)$. 
It is then easy to see that the first integral in the above identity is small in $L^{1}\left(D_{\delta}\right)$ if $\delta$ is small. For the $L^{1}\left(D_{\delta}\right)$ norm of the 2 nd integral we derive that

$$
\begin{aligned}
\int_{D \times D_{\delta}} \frac{\left|\bar{\partial} \phi_{i}\right|}{|\xi-z|} d \xi d z & =\int_{D} d \xi\left|\bar{\partial} \phi_{i}\right| \int_{D_{\delta}} \frac{d z}{|\xi-z|} \\
& \leq C \delta \int_{D}\left|\bar{\partial} \phi_{i}\right| d \xi \leq C \delta
\end{aligned}
$$

by (2.6). This shows that the $L^{1}\left(D_{\delta}\right)$-norm of $\left|\phi\left(u_{i}\right)\right|$ is small if $\delta$ is small. Since the $L^{1}$-norm of the quadrtic differential is confomally invariant, we conclude that

$$
\phi\left(v_{i}\right)=\left|\left(v_{i}\right)_{t}\right|^{2}-\left|\left(v_{i}\right)_{\theta}\right|^{2}-2 \sqrt{-1}\left(v_{i}\right)_{t} \cdot\left(v_{i}\right)_{\theta}
$$

has small $L^{1}$-norm provided $\delta$ is small.

For simplicity, we will first prove the energy identity in the case of $m=1$, i.e. $\varphi_{1}$ is the only bubble. The general case can be treated similarly, as will be explained at the end of this section. Since $m=1$, we need only to prove that $E\left(v_{i}, B_{i}\right)$ can be arbitrarily small if $\delta$ is sufficiently small and $R$ and $i$ is large enough. Given any small $\epsilon>0$, we may assume by the above results, $\delta$ is so small that

$$
\left\|\tau\left(v_{i}\right)\right\|_{L^{1}}, \quad\left\|\tau\left(v_{i}\right)\right\|_{L^{2}} \text { and }\left\|\phi\left(v_{i}\right)\right\|_{L^{1}}<\epsilon .
$$

Let $T_{0}=|\log \delta|$. Then since $v_{i} \rightarrow v_{0}=f^{*} u_{0} W^{1,2}$-strongly on $P_{M}=$ $\left[T_{0}, T_{0}+M\right] \times S^{1}$ as $i \rightarrow \infty$ for any fixed $M>0$, we have

$$
E\left(v_{i}, P_{M}\right) \rightarrow E\left(u_{0}, D_{\delta} \backslash D_{\delta e^{-M}}\right), \quad i \rightarrow \infty .
$$

We may assume that $E\left(u_{0}, D_{\delta}\right)<\epsilon / 2$ for small $\delta$. It follows that for any $M>0$ there is $i(M)$ such that

$$
E\left(v_{i}, P_{M}\right) \leq \epsilon / 2 \text { if } i \geq i(M) .
$$

Next, let $T_{i}=\left|\log \left(\lambda_{i} R\right)\right|$ and $Q_{M, i}=\left[T_{i}-M, T_{i}\right] \times S^{1}$. Similar arguments show that we may take $R$ and $i(M)$ large so that

$$
E\left(v_{i}, Q_{M, i}\right) \leq \epsilon / 2 \text { if } i \geq i(M) .
$$

Now we claim that there exits $I>0$ such that if $i \geq I$ then

$$
\int_{[t, t+1] \times S^{1}}\left|\nabla v_{i}\right|^{2}<\epsilon \text { for } t \in\left[T_{0}, T_{i}-1\right] .
$$


Suppose that the claim is false, then we may assume that as $i \rightarrow \infty$, there exist $t_{i}$ such that

$$
\int_{\left[t_{i}, t_{i}+1\right] \times S^{1}}\left|\nabla v_{i}\right|^{2} \geq \epsilon .
$$

In view of (2.8)-(2.9), we must have $t_{i}-T_{0} \rightarrow \infty$ and $T_{i}-t_{i} \rightarrow \infty$. Thus, by translation $t \rightarrow t-t_{i}$ we may consider $v_{i}$ as a map on $\left[-M_{i}, M_{i}\right] \times S^{1}$ with $M_{i} \rightarrow \infty$ and

$$
\int_{[0,1] \times S^{1}}\left|\nabla v_{i}\right|^{2} \geq \epsilon .
$$

We may assume that $v_{i} \rightarrow v_{\infty}$ weakly in $W_{l o c}^{1,2}\left(R^{1} \times S^{1}, N\right)$. Moreover, by the definition of $v_{i}$ and the assumption that $\tau\left(u_{i}\right)$ has bounded $L^{2}$-norm, it is easy to see that

$$
\left|\tau\left(v_{i}\right)\right| \rightarrow 0 \text { in } L_{l o c}^{2}\left(R^{1} \times S^{1}\right) .
$$

Now, if the convergence $v_{i} \rightarrow v_{\infty}$ is $W^{1,2}$-strong on $[0,1] \times S^{1}$, then (2.11) and (2.12) imply that $v_{\infty}$ is a nonconstant harmonic map with finite energy. Since $R^{1} \times S^{1}$ is conformal to $S^{2} \backslash\{N, S\}, v_{\infty}$ gives rise to a nonconstant harmonic sphere $\phi_{2}: S^{2} \rightarrow N$, a contradiction to the assumption that $m=1$. Next, if the convergence is not strong, then by Lemma 2.1 there exists some $p \in[0,1] \times S^{1}$ and energy concentration near the point $p$, namely along some subsequence we have

$$
\lim _{i \rightarrow \infty} E\left(v_{i}, D_{r}(p)\right) \geq \epsilon_{0}>0,
$$

for any $r>0$. In such a case we can still obtain a second harmonic sphere $\varphi_{2}$ by the rescaling argument. (Note that such an argument can go through because we have Lemma 2.1.) This shows that the claimed (2.10) must be true.

Now we remark that (2.7), (2.10) and the estimate (2.1) in Lemma 2.1, together with the imbedding $W^{2,2} \subset C^{\gamma}(\gamma \in(0,1))$ implies that

$$
\left\|v_{i}-\bar{v}_{i, t}\right\|_{C^{\gamma}\left([t, t+1] \times S^{1}, N\right)} \leq C \epsilon^{1 / 2},
$$

for each $t \in\left[T_{0}, T_{i}-1\right]$, at least for $i$ large enough. Here $\bar{v}_{i, t}$ is the mean value of $v_{i}$ over $[t, t+1] \times S^{1}$, and $C$ may depend on $\gamma$.

To estimate the energy of $v_{i}$ on $\left[T_{0}, T_{i}\right] \times S^{1}$ we employ the technique used by Sacks and Uhlenbeck in their proof of removable singularity theorem. Let $n$ be an integer such that $d=\left(T_{i}-T_{0}\right) / n \leq 1$, and let $t_{k}=T_{0}+k d$ 
for $k=0,1, \ldots, n$. We will simply write $v=v_{i}, P=\left[T_{0}, T_{i}\right] \times S^{1}, P_{k}=$ $\left[t_{k}, t_{k+1}\right] \times S^{1}$, and $S_{k}=\left\{t_{k}\right\} \times S^{1}$. Let $h(t)$ be an $R^{k}$ valued piecewise linear function $h(t)$ such that $h\left(t_{i}\right)$ equals the mean value of $u$ over $S_{i}$. Consider $h$ as a map from $R$ into $R^{K}$. Then we have

$$
\Delta(v-h)+A=\alpha,
$$

where $A=A(v)(d v, d v)$. Taking the inner product of this equation with $v-h$ and integrating over $R_{k}$ we get

$$
\int_{P_{k}}|\nabla(v-h)|^{2}=\int_{P_{k}}(v-h) \cdot(A-\alpha)+\int_{S_{k+1}}-\int_{S_{k}}(v-h) \cdot\left(v_{t}-h_{t}\right) d \theta .
$$

Note that the boundary integrals of $(v-h) \cdot h_{t}$ vanish, also that $|v-h| \leq C \sqrt{\epsilon}$ by (2.13). So we have

$$
\begin{aligned}
\int_{P_{k}}|\nabla(v-h)|^{2} \leq & C \epsilon^{1 / 2} \int_{P_{k}}|\nabla v|^{2} \\
& +C \epsilon^{1 / 2} \int_{P_{k}}|\alpha|+\int_{S_{k+1}}-\int_{S_{k}}(v-h) \cdot v_{t} d \theta .
\end{aligned}
$$

Summing the inequality over $i$ we get

$$
\begin{aligned}
\int_{P}|\nabla(v-h)|^{2} \leq & C \epsilon^{1 / 2}\left[\int_{P}|\nabla v|^{2}+\int_{P}|\alpha|\right] \\
& +\int_{S_{0}}(v-h) \cdot v_{t}-\int_{S_{n}}(v-h) \cdot v_{t} \\
\leq & C \epsilon^{1 / 2}(E(v)+C \epsilon) .
\end{aligned}
$$

Here we have used (2.7) and the fact that the integrals of $\left|v_{t}\right|$ over $S_{0}$ and $S_{n}$ are dominated by the $W^{2,2}$-norm of $v$ on a neiborghhood of the two circles, and the later is small by Lemma 2.1 (noting that $v$ has small local energy). Since $h$ is independent of $\theta$, the above estimate implies that

$$
\int_{P}\left|v_{\theta}\right|^{2} \leq C \epsilon^{1 / 2} E(v)+C \epsilon .
$$

But it is clear that $\left|v_{t}\right|^{2} \leq|\phi(v)|+\left|v_{\theta}\right|^{2}$, so we get

$$
\int_{P}\left|v_{t}\right|^{2} \leq \int_{P}|\phi(v)|+C \epsilon^{1 / 2} E(v)+C \epsilon \leq+C \epsilon^{1 / 2} E(v)+C \epsilon .
$$

Combining the above two inequalities we finally obtain

$$
E(v) \leq\left(1-2 C \epsilon^{1 / 2}\right)^{-1} C \epsilon \leq C \epsilon .
$$


This proves the energy identity for the case of $m=1$.

For the general case that $m>1$, the essential part of the proof is completely same as the above proof for $m=1$. One just has to distinguishes more "bubble domains" and "neck domains", and show that the energy on the neck domains is arbitrarily small. The proof can be completed by induction in $m$, the number of bubbles. For simplicity, we just indicate how to reduce the proof of $m=2$ to that of $m=1$.

If $m=2$, the inequality (2.10) no longer holds, and after translations we must have (2.11). Then we have two possibilities: (i) the convergence $v_{i} \rightarrow v_{\infty}$ is $W^{1,2}$-strong and $v_{\infty}$ gives rise to a nonconstant harmonic map $\varphi_{2}: S^{2} \rightarrow N$; (ii) energy of $v_{i}$ concentrates near some point $p \in[0,1] \times S^{1}$, and after suitable rescalings we get the second bubble $\varphi_{2}$. Letting $t_{i}-T_{0}=$ $A_{i}$ and $T_{i}-t_{i}=B_{i}$, we need to show that the energy of $v_{i}$ on the cylinder

$$
\left[-A_{i}, B_{i}\right] \times S^{1}
$$

can be very close to $E\left(\phi_{2}\right)$ in either case. Note that we have $A_{i}$ and $B_{i} \rightarrow \infty$ as $i \rightarrow \infty$.

In case (i), we see that since $m=2$ there is no local energy concentration, hence $v_{i} \rightarrow v_{\infty}$ in $W_{l o c}^{1,2}\left(R^{1} \times S^{1}, N\right)$. In other words, we have

$$
\lim _{M \rightarrow \infty} \lim _{i \rightarrow \infty} E\left(v_{i},[-M, M] \times S^{1}\right)=E\left(\varphi_{2}\right) .
$$

On the other hand, on the two cylinders $\left[-A_{i}, M\right] \times S^{1}$ and $\left[M, B_{i}\right] \times S^{1}$, one can show using the same method as in the proof for $m=1$ that the energy of $v_{i}$ is small provided we choose $\delta$ small and $i, R$, and $M$ large. This shows the energy identity holds.

In case (ii), we may assume that $p=(0,1)$. Then since $m=2$, there is only one bubble in $(-1,1) \times S^{1}$ for the sequence $v_{i}$. It follows from the result for $m=1$ that

$$
\lim _{i \rightarrow \infty} E\left(v_{i},(-1,1) \times S^{1}\right)=E\left(\varphi_{2}\right),
$$

while the energy of $v_{i}$ on the two cylinders $\left(-A_{i},-1\right) \times S^{1}$ and $\left(1, B_{i}\right) \times S^{1}$ can be estimated as before. Thus, we obtain the energy identity in this case too. This completes our proof of Theorem 2.

\section{Two Remarks.}

It is likely that our proof of Theorem 2 can be generalized to certain singular targets, such as Alexandrov spaces with curvature bounded from above and algebraic varieties. We hope to treat this problem in the future. 
In the case $\tau\left(u_{i}\right) \equiv 0$, i.e. $u_{i}$ is harmonic, by an asymptotic analysis (cf. [S], [CT]), we can show that the neck connecting the bubbles and $u_{0}$ in Theorem 2 converges exponentially to a geodesic in $N$. The key idea of the proof is as follows: On the neck cylinder $[-A, B] \times S^{1}$, the gradient of $u_{i}$ is pointwisely small. Therefore, one can rewrite the harmonicity equation as a perturbation of the Laplacian equation on $[-A, B] \times S^{1}$. The unperturbed linear system has two special kinds of solutions, namely constant and linear functions, both independent of the variable $\theta \in S^{1}$. These constant and linear solutions are respectively the infinitesimal form of constant maps and maps with geodesic image. Any solution perpendicular to those special ones either decays or grows exponentially. It is natural to expect that such behaviours are preserved for solutions of the perturbed equation. By an argument in $[\mathrm{CT}]$, one can then prove that connecting neck converges exponentially to a geodesic.

One can also apply the same arguments to harmonic maps from Riemann surfaces with varing conformal structure. Though energy may lose from necks connecting bubbles and the limiting map, one can show that those connecting necks converge exponentially to geodesics. Simple examples show that this is the best one can get in the general case of harmonic maps.

We expect that similar result holds true for solutions of the heat equation (1.1). Namely, if $u\left(t_{i}\right)$ are given as in Theorem 1, then the connecting neck converges to a geodesic in $N$.

\section{References.}

[CT] Cheeger, J. and Tian, G. The cone strucure of complete Ricci-flat manifolds with quadratic curvature decay, Invent. Math. 1994.

[J] Jost, J. Two-Dimensional Geometric Variational Problems, John Wiley \& Sons, Chichester, 1991.

[P] Parker, T. Bubble tree convergence for harmonic maps, preprint.

[Q] Qing, J. On singularities of the heat flow for harmonic maps from surfaces into spheres, Comm. in Analysis and Geometry, 3 (1994), 297-317.

[S] Simon, L. Asymptotics for a class of nonlinear evolution equations with applications to geometric problems, Ann. of Math. 118 (1983), 525-571.

[S-U] Sacks, J. and Uhlenbeck, K. The existence of minimal immersions of two spheres, Ann. Math. 113 (1981), 1-24. 
[St] Struwe, M. On the evolution of harmonic maps of Riemannian surfaces, Comment. Math. Helv. 60 (1985), 558-581.

RECEIVED JUNE 12TH, 1995.

INSTITUTE OF MATHEMATICS

Academia Sinica

BEJING

AND

Courant Institute of Mathematical Sciences

NEW YORK UNIVERSITY 